\title{
A case of paroxysmal kinesigenic dyskinesia which exhibited the phenotype of anxiety disorder
}

\author{
This article was published in the following Dove Press journal: \\ Neuropsychiatric Disease and Treatment \\ 16 August 2017 \\ Number of times this article has been viewed
}

\author{
Yasuto Kunii ${ }^{1,2}$ \\ Nozomu Matsuda ${ }^{3}$ \\ Hirooki Yabe' \\ 'Department of Neuropsychiatry, \\ Fukushima Medical University School \\ of Medicine, Fukushima, Japan; \\ ${ }^{2}$ Department of Neuropsychiatry, Aizu \\ Medical Center, School of Medicine, \\ Fukushima Medical University, \\ Fukushima, Japan; ${ }^{3}$ Department \\ of Neurology, Fukushima Medical \\ University School of Medicine, \\ Fukushima, Japan
}

\begin{abstract}
Background: Paroxysmal kinesigenic dyskinesia (PKD) is a rare heritable neurologic disorder characterized by attacks of involuntary movement induced by sudden voluntary movements. No previous reports have described cases showing comorbidity with psychiatric disease or symptoms. In this case, we showed a patient with PKD who exhibited several manifestations of anxiety disorder.
\end{abstract}

Case: A 35-year-old Japanese man with PKD had been maintained on carbamazepine since he was 16 years of age without any attacks. However, 10 years before this referral, he became aware of a feeling of breakdown in his overall physical functions. He had then avoided becoming familiar with people out of concern that his physical dysfunctions might be perceived in a negative light. One day he was referred by the neurologic department at our hospital to the Department of Psychiatry because of severe anxiety and hyperventilation triggered by carbamazepine. We treated with escitalopram, aripiprazole, and ethyl loflazepate. Both his subjective physical condition and objective expressions subsequently showed gradual improvement. At last, the feelings of chest compression and anxiety entirely disappeared. Accordingly, increases in plasma monoamine metabolite levels were observed, and the c.649dupC mutation, which has been found in most Japanese PKD families, was detected in his proline-rich transmembrane protein 2 gene.

Conclusion: This is the first report to describe psychiatric comorbidities or symptoms in a PDD case. The efficacy of psychotropic medication used in this case, the resulting changes in plasma monoamine metabolite levels, and the recent advances in the molecular understanding of PKD suggested slight, but widespread alterations to the neurotransmitter systems in the brain.

Keywords: paroxysmal kinesigenic dyskinesia, anxiety disorder, proline-rich transmembrane protein 2, synaptosomal-associated protein 25

\section{Introduction}

Paroxysmal kinesigenic dyskinesia (PKD) is a rare heritable neurologic disorder characterized by attacks of involuntary movement comprising any combination of chorea, ballism, athetosis, and dystonia induced by sudden voluntary movements. ${ }^{1}$ No previous reports have described cases showing comorbidity with psychiatric disease or symptoms. However, the presence of some sort of mental dysfunction in PKD patients seems highly likely, given the actions of PRRT2, which is encoded by the gene responsible for PKD. ${ }^{2}$ Although the detailed functions of PRRT2 have yet to be elucidated, this protein interacts with SNAP25, which localizes in the presynaptic terminal and is involved in the exocytosis of various neurotransmitters. ${ }^{3}$ We describe herein the case of a patient with PKD who exhibited several manifestations of anxiety disorder, including panic attacks after a change to his prescribed antiepileptic. Based on the results of plasma monoamine metabolite levels and recent advances
Correspondence: Yasuto Kunii Department of Neuropsychiatry, Fukushima Medical University, School of Medicine, I-Hikarigaoka, Fukushima 960-I295, Japan

Tel $+8 \mid 24547$ I33 |

Fax +8I 245486735

Email kunii@fmu.ac.jp 
in the molecular understanding of PKD, we discuss this case in terms of psychiatric symptoms. Written informed consent was provided by the patient to have this case report published.

\section{Case report}

A 35-year-old Japanese man with no past psychiatric history, but with a past neurologic history of PKD, was referred by the neurologic department at our hospital to the Department of Psychiatry because of severe anxiety and hyperventilation. When the patient was 16 years old, he was clinically diagnosed as PKD by a neurologist based on characteristic symptoms including involuntary movement induced by voluntary movements and various examination findings, such as test using blood and cerebrospinal fluid, and several image findings. After that, he had been maintained on carbamazepine (CBZ) at $200 \mathrm{mg}$ /day since 16 years of age without any attacks.

In February 2013, on a regular follow-up in the neurologic department, the neurologist changed CBZ to topiramate after the patient complained of long-term symptoms of drowsiness and wobbliness, which he thought might be the side effects of CBZ. Two days later, he visited the emergency department at our hospital with chest pain and hyperventilation. Although the neurologist suspended topiramate and resumed $\mathrm{CBZ}$, his anxiety and hyperventilation did not improve as was expected. The following month, the patient was referred to our department, but he stated that he had no anxiety about PKD or any other psychosocial stressors.
He presented as a gentle young man without any abnormal affect, but seemed somewhat nervous with an impassive facial appearance. We took time to have a good discussion about his history and symptoms. At last he confessed that, 10 years before this referral, he became aware of a feeling of breakdown in his overall physical functions to what he considered as about $70 \%$ of his earlier state. He had then avoided becoming familiar with people out of concern that his physical dysfunctions might be perceived in a negative light. It means that his anxiety symptoms had existed and persisted 10 years before a change of drug, CBZ to TPM, though we could not completely deny it affected these symptoms. Then we began treatment with escitalopram at $10 \mathrm{mg} /$ day, targeting the symptoms of anxiety and depression. Moreover, we added aripiprazole at $3 \mathrm{mg} /$ day and ethyl loflazepate at $1 \mathrm{mg}$ /day (Figure 1). Both his subjective physical condition and objective expressions subsequently showed gradual improvement. Feelings of chest compression and anxiety entirely disappeared. By contrast, his speed of speech and amount of conversation increased markedly, and he seemed more cheerful in his appearance. He stated, "Amazing! After 10 years' absence, this feeling came to me! My ability to judge things has been improved and I don't feel hesitant in starting new things," "I can perform complicated movements and quick motions. Now I can run and swim!," "I gained a larger circle of acquaintances and at last I have a girlfriend!." He himself called this phenomenon "Awakenings", as an analogy to the well-known movie.

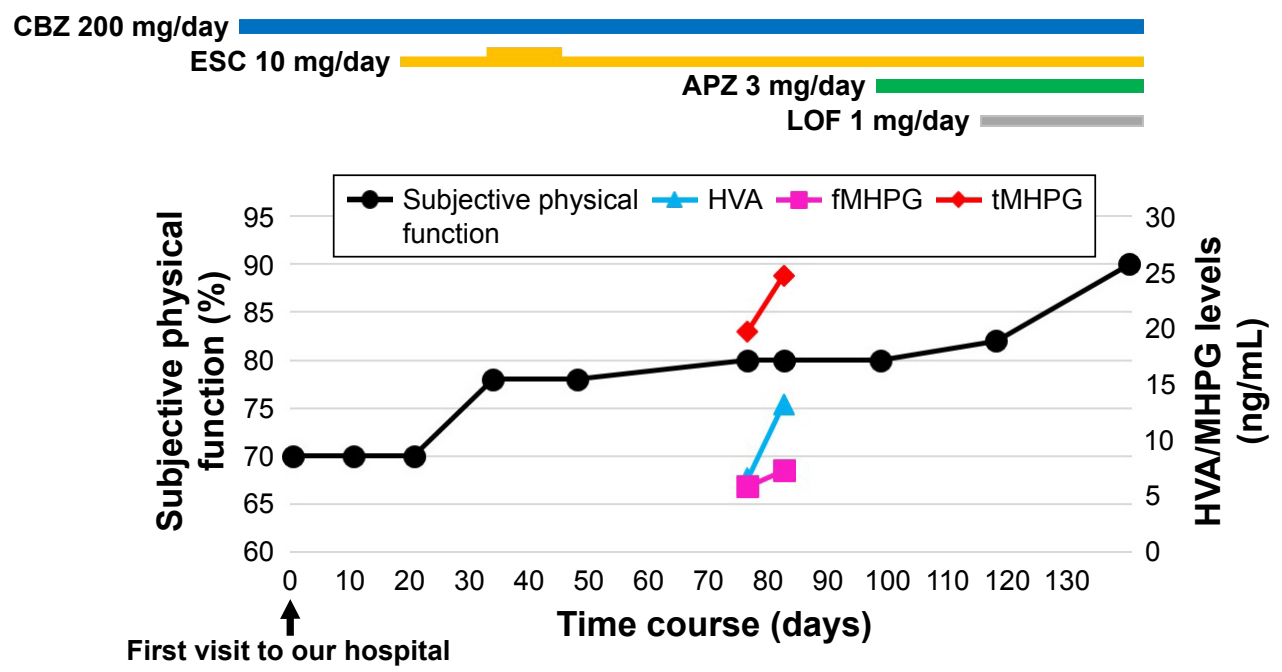

Figure I Illustration of the clinical course.

Note: Subjective overall physical functions, plasma monoamine metabolite levels (HVA [dopamine metabolite] level, total MHPG [noradrenaline metabolite] level, and free MHPG), and drugs administered to the 35-year-old man with paroxysmal kinesigenic dyskinesia (CBZ, ESC, APZ, and LOF).

Abbreviations: APZ, aripiprazole; CBZ, carbamazepine; ESC, escitalopram; HVA, homovanillic acid; LOF, loflazepate; MHPG, 3-methoxy-4-hydroxyphenylglycol; fMHPG; free MHPG, tMHPG; total MHPG. 
In short, escitalopram, aripiprazole, and ethyl loflazepate proved effective in addressing both subjective and objective symptoms (but increased doses of these drugs have loss of efficacy) in the present case. As a result, his quality of life was prominently enhanced as the scope of his activities and friendships rapidly expanded. In addition, increases in plasma monoamine metabolite levels were observed for homovanillic acid (a dopamine metabolite), total 3-methoxy4-hydroxyphenylglycol (a noradrenaline metabolite), and free 3-methoxy-4-hydroxyphenylglycol, which were analyzed with high-performance liquid chromatography with electrochemical detection. In particular, homovanillic acid level was doubled after this change in pharmacotherapy (Figure 1). Furthermore, the c.649dupC mutation, which has been found in most Japanese PKD families, ${ }^{4}$ was detected in his PRRT2 by direct sequencing after polymerase chain reaction amplification.

\section{Discussion}

This is the first report to refer to psychiatric comorbidities or symptoms in a PKD case. Although the detailed pathophysiology of PKD has yet to be clarified, recently, great advances have been achieved in the molecular understanding of PKD and surrounding areas. Genetic variations in SNAP25, the gene product of which interacts with PRRT2 encoded by the gene responsible for PKD, are reportedly associated with attention-deficit/hyperactivity disorder ${ }^{5}$ and early-onset bipolar disorder. ${ }^{6}$ Postmortem brain studies have revealed altered expressions of SNAP25 mRNA and protein in the prefrontal cortex of patients with schizophrenia ${ }^{7}$ and bipolar disorder. ${ }^{6}$ In addition, results from several imaging studies ${ }^{8,9}$ have suggested that an abnormal cortico-striato-pallidothalamic loop might underlie the pathophysiology in PKD.

In this case, we assumed that since childhood, he had fallen into a habit of suppressing overall physical activities to avoid the manifestation of involuntary movements accompanying PKD, and this habituation might have affected his mental activity and social functioning, finally resulting in anxiety disorder-like manifestations. Alternatively, PRRT2 mutation might also have been related to his shy behavior, leading to an anxiety phenotype, since SNAP 25 mutation has been reported to induce anxiety-related behavior in mice. ${ }^{10}$ Interestingly, serotonin and dopamine releases were markedly reduced in the amygdala in mutant mice in that study. The efficacy of psychotropic drugs used in this case and the resulting changes in plasma monoamine metabolite levels suggested slight, but widespread alterations to the neurotransmitter systems in the brain. This hypothesis is consistent with dysfunction of SNAP25, which is considered to be etiologically important in PKD. These findings may also provide insights into elucidating the pathophysiology of other psychiatric diseases considered to be associated with variations in SNAP25, such as schizophrenia, bipolar disorder, and attention-deficit/hyperactivity disorder.

\section{Conclusion}

In this report, we presented a patient with PKD who showed several manifestations of anxiety disorder. Based on the response to psychotropic medication and changes in plasma monoamine metabolite levels in this case, it was suggested that there might be widespread alterations to the neurotransmitter systems in the brain with PKD. Given the recent advances in the molecular understanding of PKD, these findings may also provide insights into explaining the pathophysiology of other psychiatric diseases.

\section{Acknowledgment}

We thank Dr Shinji Ono for carrying out the mutation analysis of PRRT2.

\section{Author contributions}

YK, NM, and HY designed the research and contributed to interpretations of all results; YK and NM treated the patient clinically; YK wrote the paper. All authors made substantial contributions to conception and design, acquisition of data, or analysis and interpretation of data; took part in drafting the article or revising it critically for important intellectual content; gave final approval of the version to be published; and agree to be accountable for all aspects of the work.

\section{Disclosure}

The authors report no conflicts of interest in this work.

\section{References}

1. Bruno MK, Hallett M, Gwinn-Hardy K, et al. Clinical evaluation of idiopathic paroxysmal kinesigenic dyskinesia: new diagnostic criteria. Neurology. 2004;63(12):2280-2287.

2. Wang JL, Cao L, Li XH, et al. Identification of PRRT2 as the causative gene of paroxysmal kinesigenic dyskinesias. Brain. 2011;134(Pt 12): 3493-3501.

3. Lee HY, Huang Y, Bruneau N, et al. Mutations in the gene PRRT2 cause paroxysmal kinesigenic dyskinesia with infantile convulsions. Cell Rep. 2012;1(1):2-12.

4. Ono S, Yoshiura K, Kinoshita A, et al. Mutations in PRRT2 responsible for paroxysmal kinesigenic dyskinesias also cause benign familial infantile convulsions. J Hum Genet. 2012;57(5):338-341.

5. Faraone SV, Khan SA. Candidate gene studies of attention-deficit/ hyperactivity disorder. J Clin Psychiatry. 2006;67(Suppl 8):13-20.

6. Etain B, Dumaine A, Mathieu F, et al. A SNAP25 promoter variant is associated with early-onset bipolar disorder and a high expression level in brain. Mol Psychiatry. 2010;15(7):748-755. 
7. Karson CN, Mrak RE, Schluterman KO, Sturner WQ, Sheng JG, Griffin WS. Alterations in synaptic proteins and their encoding mRNAs in prefrontal cortex in schizophrenia: a possible neurochemical basis for 'hypofrontality'. Mol Psychiatry. 1999;4(1):39-45.

8. Zhou B, Chen Q, Zhang Q, et al. Hyperactive putamen in patients with paroxysmal kinesigenic choreoathetosis: a resting-state functional magnetic resonance imaging study. Mov Disord. 2010;25(9):1226-1231.
9. Zhou B, Chen Q, Gong Q, Tang H, Zhou D. The thalamic ultrastructural abnormalities in paroxysmal kinesigenic choreoathetosis: a diffusion tensor imaging study. J Neurol. 2010;257(3):405-409.

10. Kataoka M, Yamamori S, Suzuki E, et al. A single amino acid mutation in SNAP-25 induces anxiety-related behavior in mouse. PLoS One. 2011;6(9):e25158.

\section{Publish your work in this journal}

Neuropsychiatric Disease and Treatment is an international, peerreviewed journal of clinical therapeutics and pharmacology focusing on concise rapid reporting of clinical or pre-clinical studies on a range of neuropsychiatric and neurological disorders. This journal is indexed on PubMed Central, the 'PsycINFO' database and CAS, and is the official journal of The International Neuropsychiatric Association (INA). The manuscript management system is completely online and includes a very quick and fair peer-review system, which is all easy to use. Visit http://www.dovepress.com/testimonials.php to read real quotes from published authors.

Submit your manuscript here: http://www.dovepress.com/neuropsychiatric-disease-and-treatment-journal 